Dorlan J. Kimbrough, MD

Maureen A. Mealy, RN Alexandra Simpson, MS Michael Levy, MD, PhD

Correspondence to Dr. Levy: mlevy@jhmi.edu

\section{Predictors of recurrence following an initial episode of transverse myelitis}

OPEN

ABSTRACT

Objective: This study sought to identify factors that increased the risk of recurrence after an initial transverse myelitis (TM) presentation.

Methods: Retrospective cohort study of 192 patients initially presenting with TM of unknown etiology. Patients diagnosed with multiple sclerosis during the first myelitis episode were excluded. Demographic and laboratory data were analyzed for associations with recurrence.

Results: One hundred ten of 192 patients (57\%) eventually developed recurrent symptoms: 69 (63\%) neuromyelitis optica (NMO) or NMO spectrum disorder, 34 (31\%) non-NMO recurrent $\mathrm{TM}$, and $7(6 \%)$ systemic autoimmune disease. Multiple independent risk factors for recurrence were identified: African American race (risk ratio 1.60, $p<0.001,95 \%$ confidence interval 1.26-2.03; similarly noted hereafter), female sex (1.88, $p=0.007,1.19-2.98)$, longitudinally extensive myelitis at onset $(1.34, p=0.036,1.01-1.78)$, Sjogren syndrome antigen $A(1.89, p=$ $0.003,1.44-2.48)$, vitamin $D$ insufficiency $(4.00, p<0.001,1.60-10.0)$, antinuclear antibody titer $\geq 1: 160$ (1.69, $p=0.006,1.23-2.32)$, and the presence of inflammatory markers (e.g., immunoglobulin $\mathrm{G}$ index) in the CSF $(2.14, p<0.001,1.44-3.17)$.

Conclusions: Sex, race, and serologic biomarkers warrant consideration when assessing risk of TM recurrence. Male sex and Caucasian American race were independently associated with risk of monophasic idiopathic TM. Recurrence risk in female and African American patients appears driven by a greater likelihood of developing NMO or NMO spectrum disorder. Neurol Neuroimmunol Neuroinflammation 2014;1:e4; doi: 10.1212/NXI.0000000000000004

\section{GLOSSARY}

ANA = antinuclear antibody; lg = immunoglobulin; JHH = Johns Hopkins Hospital; LETM = longitudinally extensive TM; MAD = median absolute deviation; $\mathbf{M S}=$ multiple sclerosis; $\mathbf{N M O}=$ neuromyelitis optica; $\mathbf{N M O S D}=$ NMO spectrum disorder; $\mathbf{O R}=$ odds ratio; $\mathbf{S L E}=$ systemic lupus erythematosus; $\mathbf{T M}=$ transverse myelitis.

Transverse myelitis (TM) can occur in multiple disease contexts, including multiple sclerosis (MS), neuromyelitis optica (NMO), various infections, and autoimmune rheumatologic disorders. When patients present with an initial event of TM, diagnosis of a relapsing disease has prognostic implications and guides preventive treatment. Failure to use appropriate immunomodulatory or immunosuppressive treatment may lead to unchecked relapses and long-term disability. In contrast, a patient with suspected monophasic idiopathic TM might only require acute management, symptomatic treatment, and subsequent rehabilitation rather than longterm immunosuppressive treatment. In many circumstances, however, the evaluation of an initial TM event does not yield sufficient historical, clinical, radiologic, or laboratory data to meet diagnostic criteria for an underlying condition. ${ }^{1}$ The patient with a first-time TM episode accompanied by a normal or nondiagnostic brain MRI, normal or inconclusive laboratory data, and no prior neurologic symptoms or historical suggestion of systemic illness may be diagnosed with monophasic idiopathic TM but left with apprehension about the risk of recurrence.

From the Department of Neurology, Johns Hopkins University, Baltimore, MD

Go to Neurology.org/nn for full disclosures. Funding information and disclosures deemed relevant by the authors, if any, are provided at the end of the article. The Article Processing Charge was paid by the authors.

This is an open access article distributed under the terms of the Creative Commons Attribution-Noncommercial No Derivative 3.0 License, which permits downloading and sharing the work provided it is properly cited. The work cannot be changed in any way or used commercially. 
Previous studies have identified limited biomarkers associated with recurrence. Patients with recurrent TM tend to have lower vitamin $\mathrm{D}$ levels during presentation than those with monophasic illness, suggesting an association between recurrent TM and vitamin D insufficiency. ${ }^{2}$ Anti-Ro (SS-A) antibodies have been associated with recurrent TM. ${ }^{3}$ It is well known that a first myelitis episode may simply herald the onset of MS when it is associated with typical brain lesions on MRI and abnormal CSF studies with oligoclonal banding patterns and an elevated immunoglobulin (Ig) $G$ index. ${ }^{4}$ A single longitudinally extensive TM (LETM) in the setting of a seropositive NMO-IgG test is sufficient to diagnose $\mathrm{NMO}$ spectrum disorder (NMOSD). ${ }^{5}$

Despite this progress, there is still uncertainty surrounding the prognostic implications of an initial myelitis event, and it is not clear to what extent demographic features and/or biomarker testing influence the risk of recurrence. We performed a retrospective cohort study to identify factors at the initial presentation of TM that are associated with developing recurrent neuroinflammatory disease. Features that portend recurrence may prompt consideration of empiric preventive treatment in high-risk cases. Alternatively, some patients may be spared unnecessary long-term immunosuppression if features of their case suggest a higher likelihood of monophasic disease.

METHODS Data sources and study participants. Records of patients referred to the Johns Hopkins Transverse Myelitis Center from 2005 to 2012 were reviewed for demographic and laboratory data available at the initial evaluation of TM. Referrals included patients initially treated at Johns Hopkins Hospital (JHH) and patients from a variety of academic and private hospitals subsequently referred to JHH for consultation. The patients were categorized into 2 groups: monophasic idiopathic TM (declared monophasic after at least 3 years of untreated observation) and recurrent TM of any etiology. The recurrent group was further subdivided into recurrent myelitis of unknown etiology, NMO/NMOSD (defined by Wingerchuk 2006 criteria), and autoimmune rheumatologic diagnoses. ${ }^{6-9}$ Time to recurrence is heavily skewed to the right in patients with TM, with a median of 8 months and a median absolute deviation (MAD) of 5 months. We chose to define monophasic idiopathic TM cases after at least a 3-year relapse-free period because this duration extends more than 5 times the MAD beyond the median relapse time and is therefore likely to reflect the period during which most relapses occur.

TM diagnoses were based on clinical symptoms of acute motor, sensory, or autonomic deficits with a corresponding MRI lesion. Patients diagnosed with monophasic idiopathic
TM did not have a clinical history of optic neuritis or previous neurologic deficits, did not test positive for the NMO-IgG antibody, and did not have brain MRI that was diagnostic of other conditions (e.g., MS). Brain MRI findings were considered "nonspecific" (i.e., nondiagnostic) if any noted abnormalities were not located in typical regions associated with MS (e.g., periventricular, callosal, pericallosal, juxta-cortical, or cerebellar). We defined recurrent TM as disease involving repeated inflammatory attacks of the spinal cord without symptomatic involvement of the brain; these patients did not meet diagnostic criteria for NMO or NMOSD. ${ }^{9}$ Myelitis cases without documented MRI evidence of spinal cord involvement were not included. Longitudinally extensive lesions were defined as $\geq 3$ vertebral segment spinal cord lesions on MRI. Patients diagnosed with specific disorders such as MS during the first myelitis episode were excluded.

Standard protocol approvals, registrations, and patient consents. This retrospective study was approved by the Johns Hopkins Institutional Review Board.

Statistical analyses. Statistical analyses were performed using Stata 12 (StataCorp LP, College Station, TX). Univariate logistic regression was used to determine unadjusted odds ratios (ORs) for associations between predictor variables and the risk of developing any form of recurrent myelitis (including idiopathic recurrent myelitis, NMOSD, and rheumatologic conditions), and corresponding risk ratios were determined via standard $2 \times 2$ table calculations. Similar analyses were also performed to identify associations with the risk of developing NMO or an NMOSD and with the risk of developing a rheumatologic disorder (Sjogren syndrome or systemic lupus erythematosus [SLE]). Variables were chosen a priori and included age, sex, self-identified race, the presence or absence of a longitudinally extensive lesion during the initial myelitis episode, CSF pleocytosis (white blood cell count $>5$ cells $/ \mathrm{mm}^{3}$ ), elevated CSF protein $(>60 \mathrm{mg} / \mathrm{dL}$ ), IgG index $>0.7$, positive oligoclonal band testing, vitamin D insufficiency $(25-\mathrm{OH}$ vitamin D levels $<30 \mathrm{ng} / \mathrm{mL}$ ) or deficiency $(<20 \mathrm{ng} / \mathrm{mL})$, vitamin $B_{12}$ insufficiency, an antinuclear antibody (ANA) titer $\geq 1: 160$ ( $95 \%$ of healthy patients are excluded from SLE diagnosis at this cutoff value ${ }^{10}$ ), and the presence of antibodies to double-stranded DNA, Ro (SS-A) antigen, and La (SS-B) antigen. In addition, adjusted risk ratios and ORs were calculated with respect to age, sex, race (compared as African American vs Caucasian, as these were the 2 largest groups), and the presence or absence of a longitudinally extensive spinal cord lesion at onset. Risk ratios and adjusted risk ratios were determined using standard $2 \times 2$ table calculations and log-binomial regression, respectively. ${ }^{11}$ Reported $p$ values are 2 -tailed with a significance threshold of $p<0.05$ and were calculated via $\chi^{2}$ or Fisher exact tests as appropriate.

Because the outcome of recurrent disease was not rare among individuals in this cohort, there is an expected disparity between ORs and risk ratios, with the latter being more modest and reflective of risk compared to respective reference categories.

RESULTS Case records were reviewed for $192 \mathrm{pa-}$ tients whose initial extensive evaluation of myelitis yielded insufficient evidence to confirm a specific disorder; thus, all were initially diagnosed with monophasic idiopathic TM (table 1). Among those who followed up in our clinic, 82 patients remained diagnosed with monophasic idiopathic TM as they did not develop recurrence within at least 3 years without 
Table 1 Characteristics of 192 patients who initially presented with idiopathic transverse myelitis

\begin{tabular}{|c|c|c|c|c|c|}
\hline Characteristic & Monophasic $(n=82)$ & \multicolumn{4}{|l|}{ Recurrent } \\
\hline Age at onset, y & $42.3 \pm 17.0$ & $45.1 \pm 14.8$ & $48.0 \pm 16.6$ & $54.3 \pm 11.8$ & $46.0 \pm 15.7$ \\
\hline Female & $44(53.7)$ & 65 (94.2) & $23(67.7)$ & $6(85.7)$ & 94 (85.5) \\
\hline African American & $6(7.3)$ & $38(55.1)$ & $6(17.7)$ & $4(57.1)$ & $48(43.6)$ \\
\hline Caucasian & $71(86.6)$ & $30(43.5)$ & $24(70.6)$ & $3(42.9)$ & $57(51.8)$ \\
\hline Hispanic & $1(1.2)$ & 0 & $1(2.9)$ & 0 & $1(0.9)$ \\
\hline Other race & $1(1.2)$ & 0 & $1(2.9)$ & 0 & $1(0.9)$ \\
\hline $\begin{array}{l}\text { Longitudinally extensive } \\
\text { lesion at onset }\end{array}$ & $43 / 82(52.4)$ & $46 / 63(73.0)$ & 20/32 (62.5) & $3 / 7$ (42.9) & 69/102 (67.6) \\
\hline $\begin{array}{l}\text { Time to recurrence, mo, } \\
\text { median (range), mean } \pm \text { SD }\end{array}$ & Not applicable & $9(1-267), 22 \pm 40$ & $7(1-74), 13 \pm 15$ & $5.6(2-132), 24 \pm 48$ & $8(1-267), 20 \pm 35$ \\
\hline Oligoclonal bands positive & $8 / 51(15.7)$ & 11/28 (28.2) & $7 / 19$ (36.8) & $2 / 4(50)$ & 20/51 (39.2) \\
\hline Nonspecific brain MRI findings & 15/82 (18.3) & $33 / 69$ (47.8) & 9/32 (28.1) & $3 / 7$ (42.9) & 45/108 (41.7) \\
\hline Vitamin $D<30 \mathrm{ng} / \mathrm{mL}$ & $11 / 30(36.7)$ & 21/25 (84) & 3/3 (100) & 1/1 (100) & 25/39 (86.2) \\
\hline
\end{tabular}

Abbreviations: Ig = immunoglobulin; NMO = neuromyelitis optica; NMOSD = NMO spectrum disorder; WBC = white blood cell count.

Data are mean \pm SD or $n(\%)$, unless otherwise indicated.

immunosuppressive treatment. One hundred ten patients would eventually be diagnosed with recurrent neuroinflammatory disease: 69 patients with NMO or NMOSD, 34 patients with recurrent TM, and 7 patients with rheumatologic disease (4 with Sjogren syndrome, 3 with SLE). Age did not differ between recurrent and monophasic patients. There were higher proportions of female $(p=0.007)$ and African American patients $(p<0.001)$ in the recurrent group; the sex and race disparities were particularly apparent among NMO diagnoses. Patients with recurrent myelitis were more likely to have had nonspecific brain MRI findings at onset (risk ratio 1.55, $p<0.001,95 \%$ confidence interval 1.23-1.95).
The risk ratios and ORs for associations of predictor variables with the risk of developing any form of recurrent disease are listed in tables 2 and 3. Female sex and self-identified African American ancestry were both independently associated with higher risk of developing recurrent disease, primarily as NMO. Women were nearly twice as likely to develop recurrent disease as men, and African Americans were more likely to develop recurrent TM than Caucasian Americans (table 2). Patients with LETM at onset were more likely to develop recurrent myelitis, particularly so with eventual NMO or NMOSD.

CSF pleocytosis at onset of $>5$ white blood cells/ $\mu \mathrm{L}$, positive IgG index, positive oligoclonal band

Table 2 Adjusted risk and odds ratios for associations of demographic/clinical variables

$\begin{array}{llll}\text { Variable } & \begin{array}{l}\text { Adjusted risk ratio } \\ \text { (adjusted OR) }\end{array} & p \text { Value } & 95 \% \mathrm{Cl} \\ \begin{array}{l}\text { Any recurrent TM (NMO, rheumatologic, recurrent TM) } \\ \quad \text { African American }\end{array} & 1.60(9.92) & <0.001(<0.001) & 1.26-2.03(3.55-27.7) \\ \quad \text { Female } & 1.88(4.14) & 0.007(0.001) & 1.19-2.98(1.83-9.36) \\ \quad \text { Longitudinally extensive lesion } & 1.34(1.90) & 0.036(0.037) & 1.01-1.80(1.04-3.46) \\ \text { NMO and NMOSD } & & & \\ \quad \text { African American } & 2.26(7.73) & <0.001(<0.001) & 1.57-3.26(3.32-18.0) \\ \quad \text { Female } & 4.40(10.6) & 0.003(<0.001) & 1.66-11.7(3.25-34.7) \\ \text { Longitudinally extensive lesion } & 1.62(2.74) & 0.023(0.013) & 1.07-2.45(1.24-6.10)\end{array}$

Abbreviations: $\mathrm{Cl}$ = confidence interval; $\mathrm{NMO}=$ neuromyelitis optica; NMOSD = NMO spectrum disorder; $\mathrm{OR}=$ odds ratio; $\mathrm{TM}=$ transverse myelitis. 


\begin{tabular}{|c|c|c|c|c|}
\hline Table 3 & \multicolumn{4}{|c|}{$\begin{array}{l}\text { Unadjusted risk ratios for associations of laboratory variables with risk } \\
\text { of recurrent disease }\end{array}$} \\
\hline \multicolumn{2}{|l|}{ Variable } & Risk ratio & $p$ Value & $95 \% \mathrm{Cl}$ \\
\hline \multicolumn{5}{|c|}{ Any recurrent TM (NMO, rheumatologic, recurrent TM) } \\
\hline \multicolumn{2}{|c|}{ CSF WBC $>5$ cells $/ \mu \mathrm{L}$} & 1.67 & 0.003 & $1.14-2.45$ \\
\hline \multicolumn{2}{|c|}{ CSF protein $>60 \mathrm{mg} / \mathrm{dL}$} & 1.27 & 0.152 & $0.922-1.74$ \\
\hline \multicolumn{2}{|c|}{ IgG index $>0.7$} & 2.14 & $<0.001$ & $1.44-3.17$ \\
\hline \multicolumn{2}{|c|}{ Oligoclonal bands } & 1.45 & 0.033 & $1.05-1.99$ \\
\hline \multicolumn{2}{|c|}{ Vitamin $D<30 \mathrm{ng} / \mathrm{mL}$} & 4.00 & $<0.001$ & $1.60-9.99$ \\
\hline \multicolumn{2}{|c|}{ Vitamin $\mathrm{D}<20 \mathrm{ng} / \mathrm{mL}$} & 2.38 & 0.001 & $1.54-3.69$ \\
\hline \multicolumn{2}{|c|}{ Vitamin $B_{12}<200 \mathrm{pg} / \mathrm{mL}$} & 0.54 & 0.229 & $0.157-1.86$ \\
\hline \multicolumn{2}{|c|}{ ANA $\geq 1: 160$} & 1.69 & 0.006 & $1.23-2.32$ \\
\hline \multicolumn{2}{|c|}{ Double-stranded DNA Ab } & 1.03 & 0.664 & $0.484-2.17$ \\
\hline \multicolumn{2}{|c|}{ SS-A Ab } & 1.89 & 0.003 & $1.44-2.48$ \\
\hline \multicolumn{2}{|c|}{ SS-B Ab } & 1.97 & 0.073 & $1.61-2.43$ \\
\hline \multicolumn{5}{|c|}{ NMO and NMOSD } \\
\hline \multicolumn{2}{|c|}{ CSF WBC $>5$ cells $/ \mu \mathrm{L}$} & 1.75 & 0.050 & $1.01-3.13$ \\
\hline \multicolumn{2}{|c|}{ CSF protein $>60 \mathrm{mg} / \mathrm{dL}$} & 1.36 & 0.222 & $0.832-2.22$ \\
\hline \multicolumn{2}{|c|}{$\lg G$ index $>0.7$} & 2.04 & 0.033 & $1.07-3.90$ \\
\hline \multicolumn{2}{|c|}{ Oligoclonal bands } & 1.19 & 0.347 & $0.69-2.07$ \\
\hline \multicolumn{2}{|c|}{ Vitamin $\mathrm{D}<30 \mathrm{ng} / \mathrm{mL}$} & 3.35 & 0.002 & $1.32-8.52$ \\
\hline \multicolumn{2}{|c|}{ Vitamin $\mathrm{D}<20 \mathrm{ng} / \mathrm{mL}$} & 1.65 & 0.098 & $0.934-2.91$ \\
\hline \multicolumn{2}{|c|}{ Vitamin $B_{12}<200 \mathrm{pg} / \mathrm{mL}$} & 0.422 & 0.427 & $0.064-2.76$ \\
\hline \multicolumn{2}{|c|}{ ANA $\geq 1: 160$} & 2.35 & 0.001 & $1.50-3.68$ \\
\hline \multicolumn{2}{|c|}{ Double-stranded DNA Ab } & 1.25 & 0.999 & $0.398-3.91$ \\
\hline \multicolumn{2}{|c|}{ SS-A Ab } & 2.18 & 0.013 & $1.35-3.54$ \\
\hline \multicolumn{2}{|c|}{ SS-B Ab } & 1.45 & 0.611 & $0.522-4.03$ \\
\hline
\end{tabular}

Abbreviations: $\mathrm{Ab}=$ antibody; $\mathrm{ANA}=$ antinuclear antibody; $\mathrm{Cl}=$ confidence interval; $\mathrm{lg}=$ immunoglobulin; NMO = neuromyelitis optica; NMOSD = NMO spectrum disorder; $\mathrm{TM}=$ transverse myelitis; WBC $=$ white blood cell count.

testing, vitamin $\mathrm{D}$ insufficiency and deficiency (25-OH vitamin $\mathrm{D}<30 \mathrm{ng} / \mathrm{mL}$ and $<20 \mathrm{ng} / \mathrm{mL}$, respectively), ANA titer of $\geq 1: 160$, and presence of antibodies to Ro/SS-A antigen were all also independently associated with increased risk of developing recurrent myelitis (table 3). Age, elevated CSF protein, vitamin $B_{12}$ insufficiency, antibodies to La/SS-B antigen, and positive double-stranded DNA antibody testing at TM onset were not associated with recurrent disease in this cohort.

Eventual diagnoses of NMO or NMOSD accounted for 69 of the 110 recurrent myelitis cases (62.7\%). More specifically, 24 of these 69 cases (35\%) were diagnosed with NMO and eventually tested positive for NMO IgG at some point after the initial presentation; 7 cases (10\%) met diagnostic criteria for NMO despite negative NMO IgG tests, and 38 cases $(55 \%)$ were diagnosed as NMOSD. The risk ratios and ORs for associations of predictor variables with the risk of developing $\mathrm{NMO}$ or an
NMOSD are listed in tables 2 and 3. Features associated with eventual diagnosis of NMO/NMOSD included LETM at onset, female sex, African American race, elevated IgG index, vitamin D insufficiency, an ANA titer of $\geq 1: 160$, and antibodies to Ro/SS-A antigen.

DISCUSSION In the cohort under study, female and African American patients had an increased risk of recurrence following a first myelitis event for which initial evaluation yielded insufficient evidence to confirm a specific disorder. The risk of recurrence in these groups appears to be driven by their greater likelihood of developing NMO or NMOSD. Male and Caucasian American patients were more likely to be diagnosed with monophasic idiopathic TM (defined herein as at least 3 years without a relapse). Patients with an ANA titer of $\geq 1: 160$ had increased risk of recurrence, which typically occurred in the context of NMO, Sjogren syndrome, or SLE. This study also supports previously reported associations between the risk of recurrent TM and vitamin D insufficiency, antiRo/SS-A antibodies, and evidence of inflammation in the CSF during the initial episode. In addition, the results support the notion that while LETM is often associated with recurrence in the context of $\mathrm{NMO} /$ NMOSD, it can occur in monophasic cases and non-NMO recurrent TM cases as well. ${ }^{12}$ In addition to NMO, idiopathic monophasic TM, and nonNMO recurrent TM, the differential diagnosis of longitudinally extensive myelitis encompasses various conditions, including Sjogren syndrome, SLE, sarcoidosis, neuro-Behçet disease, and parainfectious myelitis. ${ }^{13-16}$

There are varied reports of the sex distribution across all forms of myelitis. Several studies observe a female preponderance among patients with $\mathrm{NMO}$, including sex ratios of 10:1, 6.5:1, and 3:1 in Japan, the United States, and France, respectively. ${ }^{17-20}$ Reports of the sex distribution in non-NMO recurrent myelitis, however, are somewhat disparate. For example, while the Transverse Myelitis Consortium Working Group noted equal incidence of acute TM in men and women, there was a preponderance of male patients in a series of South Korean idiopathic recurrent TM cases. ${ }^{21,22}$ Also, among a cohort of 13 patients with idiopathic myelitis in an Italian study, 4 relapsed, all of whom were men. ${ }^{23}$ In contrast, there was a markedly higher incidence of TM in women compared to men among a Northern California population, although it was not clear what proportion of these patients may have had other features conferring risk of an eventual MS diagnosis. ${ }^{24}$

Reports of the racial and ethnic distribution of TM are varied as well. No particular ethnic or racial predisposition has been noted regarding idiopathic 
TM. ${ }^{25-28}$ Among patients of African or East Asian ancestry with demyelinating illness, NMO is a common diagnosis in a considerable number of case series around the world, including series in Japan, Great Britain, Nigeria, and Martinique. ${ }^{29-32}$ In our analysis, which included 54 African Americans, 48 patients $(88.9 \%)$ relapsed, while only $6(11.1 \%)$ remained monophasic. In contrast, out of 128 Caucasian Americans, just 57 patients (44.5\%) relapsed.

Among patients whose initial workup discloses no diagnosis more specific than idiopathic TM (e.g., a brain MRI without lesions, no current or historical optic neuritis), it may be possible to better anticipate who will develop recurrent events. Multiple independent risk factors represent considerations to be weighed in clinical circumstances. The presence of CSF abnormalities, vitamin D insufficiency, and serum autoantibodies-particularly NMO-IgG, anti-Ro/SS-A, and high titer ANA—should prompt attention to the risk of recurrence. In our study, male sex and Caucasian ancestry are more associated with monophasic TM, although this certainly does not preclude the possibility that any individual patient may have a recurrence. A full evaluation and clinical vigilance are warranted for all patients.

A potentially more controversial issue concerns whether combinations of risk factors should encourage early empiric treatment with immunosuppression. Such clinical decision-making is highly individualized, and there are no relevant clinical trials on this matter. Weighing the risk of potentially devastating recurrent TM against the risk of immunosuppression-related side effects is fraught with potential complications. In addition to considering a higher index of suspicion in patients with multiple recurrence risk factors, it may be prudent to revisit the evaluation of patients with newly diagnosed "idiopathic" TM frequently within the first 6-12 months. Serologic tests, particularly anti-aquaporin-4 antibodies, ANA, and antibodies to Ro/SS-A antigen, may yield actionable results when repeated. For example, there is evidence that anti-aquaporin- 4 antibody titers fluctuate and can become detectable before recurrence; we recommend repeating testing yearly for high-risk patients. ${ }^{33}$ There are no randomized trials showing that vitamin D supplementation mitigates the risk of recurrent TM, but supplementation may be a reasonable and benign intervention in some patients with insufficient or deficient levels.

Some limitations of this study are inherent due to its retrospective design. During the referral process, some patients with mild or relatively uncomplicated myelitis who otherwise might have been referred to the Johns Hopkins Transverse Myelitis Center may instead have been managed locally or referred to other clinics to rule out other systemic conditions based on concerns of the referring physician. There may be a more general selection bias in this study, as the examined population is comprised of patients referred to a tertiary care center and does not necessarily represent the population at large. Also, our cohort includes few patients of Asian or Hispanic ethnicities, which prevents pertinent analysis relative to and/or specific to these patient populations. Furthermore, variables were chosen for evaluation a priori, though data collection relied on retrospective review of medical chart data rather than prospective collection. Similarly, MRI was not systematically arranged prospectively, which would have been of interest in tracking the evolution or development of any associated lesions.

Inferences about results from this study may also be tempered by the possibility that some patients currently considered monophasic may eventually develop recurrent disease, and some patients currently diagnosed with recurrent TM of unknown etiology may later receive a definitive diagnosis. Accordingly, there is a continued need to better understand how recurrence risk can be determined earlier and more precisely for patients with TM.

\section{AUTHOR CONTRIBUTIONS}

D.J.K., M.A.M., and M.L. conceptualized and designed the study. D.J.K., M.A.M., A.S., and M.L. each performed data analysis and contributed to interpretation of the results. D.J.K. drafted the manuscript and all authors substantively revised the document for intellectual content.

\section{STUDY FUNDING}

No targeted funding reported.

\section{DISCLOSURE}

Dr. Kimbrough is supported by the NIH T32 Training Program in Neuroimmunology and Neuroinfectious Diseases at Johns Hopkins University, has received educational grant support from Teva Pharmaceuticals, and has received consulting fees from Medical Logix, LLC for the development of continuing medical education programs. Maureen A. Mealy has served on an advisory board for Novartis. Alexandra Simpson reports no disclosures. Dr. Levy receives research support from the NIH, Guthy Jackson Charitable Foundation, Acorda, Sanofi, NeuralStem, ApoPharma Inc., Viropharma (Shire), and Genentech; is on the scientific advisory board for Asterias; has received travel funding from Asterias and Guthy Jackson Charitable Foundation; receives publishing royalties from Lippincott Williams \& Wilkins; and serves as a consultant for Chugai Pharmaceuticals, Glaxo-Smith-Kline, and Medimmune. Go to Neurology.org/nn for full disclosures.

Received March 11, 2014. Accepted in final form March 17, 2014.

\section{REFERENCES}

1. de Seze J, Lanctin C, Lebrun C, et al. Idiopathic acute transverse myelitis: application of the recent diagnostic criteria. Neurology 2005;65:1950-1953.

2. Mealy MA, Newsome S, Greenberg BM, Wingerchuk D, Calabresi P, Levy M. Low serum vitamin D levels and recurrent inflammatory spinal cord disease. Arch Neurol 2012;69:352-356. 
3. Hummers LK, Krishnan C, Casciola-Rosen L, et al. Recurrent transverse myelitis associates with anti-Ro (SSA) autoantibodies. Neurology 2004;62:147-149.

4. Sellner J, Luthi N, Buhler R, et al. Acute partial transverse myelitis: risk factors for conversion to multiple sclerosis. Eur J Neurol 2008;15:398-405.

5. Wingerchuk DM, Lennon VA, Lucchinetti CF, Pittock SJ, Weinshenker BG. The spectrum of neuromyelitis optica. Lancet Neurol 2007;6:805-815.

6. Hochberg MC. Updating the American College of Rheumatology revised criteria for the classification of systemic lupus erythematosus. Arthritis Rheum 1997;40:1725.

7. Petri M, Orbai AM, Alarcon GS, et al. Derivation and validation of the Systemic Lupus International Collaborating Clinics classification criteria for systemic lupus erythematosus. Arthritis Rheum 2012;64:2677-2686.

8. Vitali C, Bombardieri S, Jonsson R, et al. Classification criteria for Sjogren's syndrome: a revised version of the European criteria proposed by the American-European Consensus Group. Ann Rheum Dis 2002;61:554-558.

9. Wingerchuk DM, Lennon VA, Pittock SJ, Lucchinetti CF, Weinshenker BG. Revised diagnostic criteria for neuromyelitis optica. Neurology 2006;66:1485-1489.

10. Tan EM, Feltkamp TE, Smolen JS, et al. Range of antinuclear antibodies in "healthy" individuals. Arthritis Rheum 1997;40:1601-1611.

11. McNutt LA, Wu C, Xue X, Hafner JP. Estimating the relative risk in cohort studies and clinical trials of common outcomes. Am J Epidemiol 2003;157:940-943.

12. Sepulveda M, Blanco Y, Rovira A, et al. Analysis of prognostic factors associated with longitudinally extensive transverse myelitis. Mult Scler 2013;19:742-748.

13. Habek M, Adamec I, Pavlisa G, Brinar VV. Diagnostic approach of patients with longitudinally extensive transverse myelitis. Acta Neurol Belgica 2012;112:39-43.

14. Kitley JL, Leite MI, George JS, Palace JA. The differential diagnosis of longitudinally extensive transverse myelitis. Mult Scler 2012;18:271-285.

15. Larik A, Chiong Y, Lee LC, Ng YS. Longitudinally extensive transverse myelitis associated with dengue fever. BMJ Case Rep 2012;2012.

16. White C, Leonard B, Patel A. Longitudinally extensive transverse myelitis: a catastrophic presentation of a flareup of systemic lupus erythematosus. CMAJ 2012;184: E197-E200.

17. Collongues N, Marignier R, Zephir H, et al. Neuromyelitis optica in France: a multicenter study of 125 patients. Neurology 2010;74:736-742.
18. Jacob A, McKeon A, Nakashima I, et al. Current concept of neuromyelitis optica (NMO) and NMO spectrum disorders. J Neurol Neurosurg Psychiatry 2013;84:922-930.

19. Mealy MA, Wingerchuk DM, Greenberg BM, Levy M. Epidemiology of neuromyelitis optica in the United States: a multicenter analysis. Arch Neurol 2012;69:1176-1180.

20. Nagaishi A, Takagi M, Umemura A, et al. Clinical features of neuromyelitis optica in a large Japanese cohort: comparison between phenotypes. J Neurology Neurosurgery Psychiatry 2011;82:1360-1364.

21. Transverse Myelitis Consortium Working Group. Proposed diagnostic criteria and nosology of acute transverse myelitis. Neurology 2002;59:499-505.

22. Kim KK. Idiopathic recurrent transverse myelitis. Arch Neurol 2003;60:1290-1294.

23. Ravaglia S, Bastianello S, Franciotta D, et al. NMO-IgGnegative relapsing myelitis. Spinal Cord 2009;47:531-537.

24. Klein NP, Ray P, Carpenter D, et al. Rates of autoimmune diseases in Kaiser Permanente for use in vaccine adverse event safety studies. Vaccine 2010;28:1062-1068.

25. Awad A, Stuve O. Idiopathic transverse myelitis and neuromyelitis optica: clinical profiles, pathophysiology and therapeutic choices. Curr Neuropharmacology 2011;9:417-428.

26. Christensen PB, Wermuth L, Hinge HH, Bomers K. Clinical course and long-term prognosis of acute transverse myelopathy. Acta Neurol Scand 1990;81:431-435.

27. Jeffery DR, Mandler RN, Davis LE. Transverse myelitis. Retrospective analysis of 33 cases, with differentiation of cases associated with multiple sclerosis and parainfectious events. Arch Neurol 1993;50:532-535.

28. Krishnan C, Kaplin AI, Deshpande DM, Pardo CA, Kerr DA. Transverse Myelitis: pathogenesis, diagnosis and treatment. Front Biosci 2004;9:1483-1499.

29. Cabre P, Heinzlef O, Merle H, et al. MS and neuromyelitis optica in Martinique (French West Indies). Neurology 2001;56:507-514.

30. Kira J. Multiple sclerosis in the Japanese population. Lancet Neurol 2003;2:117-127.

31. Osuntokun BO. The pattern of neurological illness in tropical Africa. Experience at Ibadan, Nigeria. J Neurol Sci 1971;12:417-442.

32. Storoni M, Pittock SJ, Weinshenker BG, Plant GT. Optic neuritis in an ethnically diverse population: higher risk of atypical cases in patients of African or African-Caribbean heritage. J Neurol Sci 2012;312:21-25.

33. Takahashi T, Fujihara K, Nakashima I, et al. Anti-aquaporin- 4 antibody is involved in the pathogenesis of NMO: a study on antibody titre. Brain A J Neurol 2007;130:1235-1243. 


\section{Neurology \\ Neuroimmunology \& Neuroinflammation}

\section{Predictors of recurrence following an initial episode of transverse myelitis \\ Dorlan J. Kimbrough, Maureen A. Mealy, Alexandra Simpson, et al. \\ Neurol Neuroimmunol Neuroinflamm 2014;1; \\ DOI 10.1212/NXI.0000000000000004}

This information is current as of April 24, 2014

\section{Updated Information \& Services}

References

Citations

Permissions \& Licensing

Reprints including high resolution figures, can be found at:

http://nn.neurology.org/content/1/1/e4.full.html

This article cites 32 articles, 4 of which you can access for free at: http://nn.neurology.org/content/1/1/e4.full.html\#\#ref-list-1

This article has been cited by 1 HighWire-hosted articles: http://nn.neurology.org/content/1/1/e4.full.html\#\#otherarticles

Information about reproducing this article in parts (figures,tables) or in its entirety can be found online at: http://nn.neurology.org/misc/about.xhtml\#permissions

Information about ordering reprints can be found online: http://nn.neurology.org/misc/addir.xhtml\#reprintsus

Neurol Neuroimmunol Neuroinflamm is an official journal of the American Academy of Neurology.

Published since April 2014, it is an open-access, online-only, continuous publication journal. Copyright $\odot$ 2014 American Academy of Neurology. All rights reserved. Online ISSN: 2332-7812.

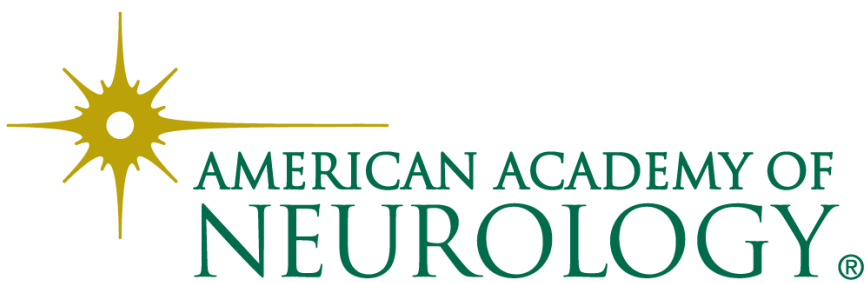

